\title{
MIXED BOUNDARY-VALUE PROBLEMS IN POTENTIAL THEORY
}

\author{
by A. H. ENGLAND \\ (Received 8th August 1977)
}

\section{Introduction}

The problems associated with finding solutions of Laplace's equation subject to mixed boundary conditions have attracted much attention and, as a consequence, a variety of analytical techniques have been developed for the solution of such problems. Sneddon (1) has given a comprehensive account of these techniques. The object of this note is to draw attention to some simple orthogonal polynomial solutions to the most basic mixed boundary-value problems in two and threedimensional potential theory. These solutions have the advantage that most quantities of physical interest are easily evaluated in terms of known functions. Two-dimensional problems are considered in $\$ 2$ and axially-symmetric three-dimensional problems in $\$ 3$.

\section{Half-plane problems}

In this section solutions are given to the two-dimensional Laplace's equation in the half-plane $y \geqslant 0$, subject to mixed boundary conditions defined on $|x|<1$ and $|x|>1$ of $y=0$. If we use the complex variable $z=x+i y$ and define the complex functions

$$
\begin{aligned}
& R_{n}(z)=\left\{z-\left(z^{2}-1\right)^{1 / 2}\right\}^{n} \quad \text { for } n>0, R_{0}(z)=1, \\
& G_{n}(z)=\frac{1}{n+1} R_{n+1}^{\prime}(z)=-\frac{R_{n+1}(z)}{\left(z^{2}-1\right)^{1 / 2}}, \quad n \geqslant-1,
\end{aligned}
$$

the solutions to the simplest mixed boundary-value problems can be expressed in terms of these functions. The functions $R_{n}(z), G_{n}(z)$ are holomorphic in the complex plane cut along $y=0,|x| \leqslant 1$ and are generalisations of the Chebyshev polynomials. In particular on the cut they satisfy

$$
\begin{gathered}
R_{n}^{ \pm}(x)=T_{n}(x) \mp i\left(1-x^{2}\right)^{1 / 2} U_{n-1}(x), \\
G_{n}^{ \pm}(x)= \pm i R_{n+1}^{ \pm}(x) /\left(1-x^{2}\right)^{1 / 2} .
\end{gathered}
$$

These functions result from generalisations of integrals given in Abramowitz and Stegun (2) and Erdogan, Gupta and Cook (3). They have been derived by Gladwell and England (4) and used in the solution of some simple elastostatic problems.

The following list gives solutions to boundary-value problems for the harmonic functions $\phi, \theta, \chi, \psi$ defined in $y \geqslant 0$, which are bounded as $|z| \rightarrow \infty$, and satisfy the 
stated conditions on $y=0$. The values of the solutions and their normal derivatives on the boundary are also given.

Problem $A$. On $y=0, \phi=T_{n}(x)$ for $|x| \leqslant 1, \partial \phi \mid \partial y=0$ for $|x| \geqslant 1$.

Solution: $\quad \phi=\operatorname{Re}\left\{R_{n}(z)\right\} \quad$ for $n \geqslant 0$.

$$
\text { On } \begin{aligned}
y=0, \frac{\partial \phi}{\partial y} & =-\frac{n T_{n}(x)}{\left(1-x^{2}\right)^{1 / 2}}, \quad|x| \leqslant 1, \\
\phi & =\left\{x-\left(x^{2}-1\right)^{1 / 2}\right\}^{n}, \quad x>1 .
\end{aligned}
$$

Problem B. On $y=0, \psi=U_{n}(x)$ for $|x| \leqslant 1, \partial \psi \mid \partial y=0$ for $|x| \geqslant 1$.

Solution: $\quad \psi=\operatorname{Re}\left\{G_{n}(z)\right\}=\operatorname{Re}\left\{\frac{1}{n+1} R_{n+1}^{\prime}(z)\right\}$ for $n \geqslant 0$,

homogeneous solution:

$$
\begin{gathered}
\psi=\operatorname{Re}\left\{G_{-1}(z)\right\}=\operatorname{Re}\left\{\left(z^{2}-1\right)^{-1 / 2}\right\} \\
\text { On } y=0: \frac{\partial \psi}{\partial y}=-\frac{(n+1) U_{n}(x)}{\left(1-x^{2}\right)^{1 / 2}}-\frac{x T_{n+1}(x)}{\left(1-x^{2}\right)^{3 / 2}} \text { for }|x|<1 \\
\psi \\
\psi=-\left\{x-\left(x^{2}-1\right)^{1 / 2}\right\}^{n+1} /\left(x^{2}-1\right)^{1 / 2} \text { for } x>1
\end{gathered}
$$

The homogeneous solution may be used to remove the boundary singularities in $\psi$ at $|x|=1$ and the singularities of order $\frac{3}{2}$ in $\partial \psi / \partial y$ at $|x|=1$ when $n$ is odd.

Problem C. On $y=0, \partial \theta / \partial y=T_{n}(x)$ for $|x| \leqslant 1, \theta=0$ for $|x| \geqslant 1$.

Solution:

$$
\begin{aligned}
& \theta=\left\{\begin{array}{l}
\operatorname{Im}\left\{\frac{1}{2(n+1)} R_{n+1}(z)-\frac{1}{2(n-1)} R_{n-1}(z)\right\} \text { for } n>1, \\
\operatorname{Im}\left\{\frac{1}{4} R_{2}(z)\right\} \quad \text { for } n=1, \\
\operatorname{Im}\left\{R_{1}(z)\right\} \quad \text { for } n=0 .
\end{array}\right. \\
& \text { On } y=0, \theta=-\frac{\left(1-x^{2}\right)^{1 / 2}}{2}\left\{\frac{U_{n}(x)}{n+1}-\frac{U_{n-2}(x)}{n-1}\right\}, \quad|x| \leqslant 1, \\
& \qquad \frac{\partial \theta}{\partial y}=-\left\{x-\left(x^{2}-1\right)^{1 / 2}\right\}^{n}, \quad x>1 .
\end{aligned}
$$

Problem D. On $y=0, \partial \chi / \partial y=U_{n}(x)$ for $|x| \leqslant 1, \chi=0$ for $|x| \geqslant 1$.

Solution: $\quad \chi=\operatorname{Im}\left\{\frac{1}{n+1} R_{n+1}(z)\right\}, \quad n \geqslant 0$.

$$
\text { On } \begin{aligned}
y=0, \chi & =-\frac{\left(1-x^{2}\right)^{1 / 2} U_{n}(x)}{n+1}, \quad|x| \leqslant 1, \\
\frac{\partial \chi}{\partial y} & =-\frac{\left\{x-\left(x^{2}-1\right)^{1 / 2}\right\}^{n+1}}{\left(x^{2}-1\right)^{1 / 2}}, \quad x>1 .
\end{aligned}
$$


Note that these problems are inter-related by the differential and recurrence relations between the Chebyshev polynomials.

The solutions to problems A, B, C and D may be derived by using elliptical polar coordinates but do not appear to be well-known. To illustrate their use consider the harmonic function $V$ defined in the half-plane $y \geqslant 0$ subject to

$$
V=g(x) \text { for }|x| \leqslant 1, \quad \partial V / \partial y=0 \text { for }|x|>1 \text { on } y=0 .
$$

If $g(x)$ is expanded in a series of Chebyshev polynomials

$$
g(x)=\sum_{0}^{\infty} a_{n} T_{n}(x) \text { in }|x| \leqslant 1
$$

the general solution is

$$
V=\sum_{0}^{\infty} a_{n} \operatorname{Re}\left\{R_{n}(x)\right\}
$$

This solution is bounded in the half-plane $y \geqslant 0$ but $\partial \psi / \partial y$ has a singularity of order $\frac{1}{2}$ at $( \pm 1,0)$. The constants $a_{n}$ may be determined by using the orthogonality relations for the Chebyshev polynomials and are

$$
a_{n}=\gamma_{n} \int_{-1}^{1} \frac{T_{n}(x)}{\left(1-x^{2}\right)^{1 / 2}} g(x) d x=\gamma_{n} \int_{0}^{\pi} \cos n \theta \quad g(\cos \theta) d \theta
$$

where $\gamma_{n}=2 / \pi, n>0$ and $\gamma_{0}=1 / \pi$.

The expansion (14) is a compact and accurate method of approximating a wellbehaved function and it is interesting to note that such series arise naturally in these problems. These series representations are discussed by Fox and Parker (5). If $g(x)$ is a polynomial, then the coefficients $a_{n}$ may be found directly from (2, Table 22.3). Alternatively the integrals may be evaluated numerically by using the Gauss-Chebyshev integration formula $(2,25.4 .38)$ which gives an accurate means of determining the constants $a_{n}$. Once this set of constants has been found, the boundary values of the potential $V$ or $\partial V / \partial y$ may be found directly from the solution to problem $A$.

It is interesting to note that if the constants $a_{n}$ are expressed in terms of the Gauss-Chebyshev integration formula $(2,25.4 .38)$ and this approximation is substituted into the form for $V$, then the following result is produced after some manipulation:

$$
V=\operatorname{Re}\left[\frac{1}{N} \sum_{r=1}^{N} \frac{g\left(x_{r}\right)}{z-x_{r}}\left\{\left(z^{2}-1\right)^{1 / 2}-(-1)^{r}\left(1-x_{r}^{2}\right)^{1 / 2} R_{N}(z)\right\}\right]
$$

where $x_{r}=\cos (2 r-1) \pi / 2 N$. Note that these expressions are not singular at $z=x_{r}$. However, it should be remarked that it is more efficient to determine the constants $a_{n}$ and to use the expansion (15) for $V$ rather than (16).

If it is required that the potential $V$ satisfying (13) tends to zero as $|z| \rightarrow \infty$, then either $g(x)$ must be such that the coefficient $a_{0}$ in (14) is zero which implies

$$
\int_{-1}^{1} \frac{g(x)}{\left(1-x^{2}\right)^{1 / 2}} d x=0
$$

or an alternative solution corresponding to $g(x)=a_{0}$ must be employed. This solution 
is given by problem $B$ with $n=0$ and is

$$
\psi=-a_{0} \operatorname{Re}\left[\frac{\left\{z-\left(z^{2}-1\right)^{1 / 2}\right\}^{2}}{\left(z^{2}-1\right)^{1 / 2}}\right] .
$$

This solution is singular on the boundary $y=0$.

A similar procedure may be applied to determine the harmonic function $W$ defined in $y \geqslant 0$ and subject to

$$
\frac{\partial W}{\partial y}=h(x) \text { for }|x| \leqslant 1, \quad W=0 \text { for }|x| \geqslant 1 \text { on } y=0 .
$$

If a representation of the form of (14) is taken for $h(x)$ then the solution may be expressed using the results of problem $C$. A more convenient representation is given by putting

$$
h(x)=\sum_{0}^{\infty} b_{n} U_{n}(x), \quad|x| \leqslant 1
$$

for then, from problem $D$,

$$
W=-\sum_{0}^{\infty} \frac{b_{n}}{n+1} \operatorname{Im}\left\{R_{n+1}(z)\right\} .
$$

This solution is bounded and tends to zero as $|z| \rightarrow \infty$. From (18) the coefficients $b_{n}$ are given by

$$
b_{n}=\frac{2}{\pi} \int_{-1}^{1}\left(1-x^{2}\right)^{1 / 2} U_{n}(x) h(x) d x=\frac{2}{\pi} \int_{0}^{\pi} \sin (n+1) \theta h(\cos \theta) \sin \theta d \theta .
$$

The representation (18) for a well-behaved function $h(x)$ is a rapidly convergent series which has similar properties to the expansion (14). If $h(x)$ is a polynomial these coefficients may be found from (2, Table 22.3). Alternatively the integrals may be approximated by using the Gauss-Chebyshev integration formulae $(2,25.4 .40)$. If this formula is used to approximate $b_{n}$, then after some manipulation

$$
\frac{\partial W}{\partial x}=\operatorname{Im}\left[\frac{1}{N+1} \sum_{r=1}^{N} \frac{h\left(x_{r}\right)\left(1-x_{r}^{2}\right)\left\{1-(-1)^{r} R_{N+1}(z)\right\}}{\left(z-x_{r}\right)\left(z^{2}-1\right)^{1 / 2}}\right]
$$

where $x_{r}=\cos r \pi /(N+1)$. Note that the terms of (20) are not singular at $z=x_{r}$. Again it should be remarked that it is more efficient to evaluate the constants $b_{n}$ and to use the solution (19) rather than (20).

It is possible to solve these problems by converting them into singular equations of the type

$$
\frac{1}{\pi} \int_{-1}^{1} \frac{f(t)}{t-x} d t=g(x) \quad \text { for }|x| \leqslant 1 .
$$

In particular for Problem $A$ where $g(x)=T_{n}(x)$ the solution is

$$
f(t)=-\left(1-t^{2}\right)^{1 / 2} U_{n-1}(t) \quad \text { for } n \geqslant 1 .
$$

There is no bounded solution of $(21)$ in the case when $g(x)$ is constant.

For Problem $B$, when $g(x)=U_{n}(x)$ the solution is 


$$
f(t)=T_{n+1}(t) /\left(1-t^{2}\right)^{1 / 2} \quad \text { for } n \geqslant 0
$$

and the solution of the homogeneous case is $f(t)=\left(1-t^{2}\right)^{-1 / 2}$. In fact,

$$
R_{n}(z)=-\frac{1}{\pi} \int_{-1}^{1} \frac{\left(1-t^{2}\right)^{1 / 2} U_{n-1}(t)}{t-z} d t, \quad n>0
$$

and

$$
G_{n}(z)=\frac{1}{\pi} \int_{-1}^{1} \frac{T_{n+1}(t)}{\left(1-t^{2}\right)^{1 / 2}(t-z)} d t, \quad n \geqslant-1 .
$$

These are extensions of the integrals given in $(2,822.13)$ which lead to the solutions (22) and (23). Problems C and D only lead to solutions of the integral equation which relate directly to (22) and (23).

Clearly representations of the types (14) or (18) may be used to construct solutions to the integral equation (21). Since the solution (22) excludes the case of a constant on the right-hand side then, when $g(x)$ has the representation (14), the solution is

$$
f(t)=-\sum_{1}^{N} a_{n}\left(1-t^{2}\right)^{1 / 2} U_{n-1}(t)+\frac{a_{0} t}{\left(1-t^{2}\right)^{1 / 2}}+\frac{B}{\left(1-t^{2}\right)^{1 / 2}},
$$

where $B$ is an arbitrary constant.

\section{Axially-symmetric potential problems}

Axially-symmetric problems may be expressed in either cylindrical polar coordinates $(\rho, \phi, z)$ or oblate spheroidal coordinates $\eta, \phi, \xi$ where

$$
\rho^{2}=\left(1-\eta^{2}\right)\left(1+\xi^{2}\right), \quad z=\eta \xi .
$$

In oblate spheroidal coordinates an axially-symmetric solution of Laplace's equation is

$$
P_{n}(\eta) Q_{n}(i \xi)
$$

where $P_{n}$ is the Legendre polynomial of degree $n$ and $Q_{n}$ is the Legendre function of the second kind. These solutions lead directly to the following mixed boundary-value problems for the harmonic functions $\chi$ and $\psi$ defined in the half-space $z \geqslant 0$ subject to the stated conditions on $z=0$.

Problem A. On $z=0, \chi=P_{2 m}\left\{\left(1-\rho^{2}\right)^{1 / 2}\right\}$ for $0 \leqslant \rho \leqslant 1, \partial \chi / \partial z=0$ for $\rho>1$.

Solution: $\quad \chi=P_{2 m}(\eta) Q_{2 m}(i \xi) / Q_{2 m}(+i 0)=\frac{2 i P_{2 m}(\eta) Q_{2 m}(i \xi)}{\pi P_{2 m}(0)}$

where $P_{2 m}(0)=(-1)^{m}(2 m) ! /\left[2^{m} m !\right]^{2}$.

$$
\begin{gathered}
\text { On } z=0, \frac{\partial \chi}{\partial z}=\frac{2 P_{2 m}\left\{\left(1-\rho^{2}\right)^{1 / 2}\right\}}{\pi\left\{P_{2 m}(0)\right\}^{2}\left(1-\rho^{2}\right)^{1 / 2}}, \quad 0 \leqslant \rho<1, \\
\chi=\frac{2}{\pi} i Q_{2 m}\left\{i\left(\rho^{2}-1\right)^{1 / 2}\right\}, \quad \rho>1 .
\end{gathered}
$$


Note that on $z=0$,

$$
\lim _{\rho \rightarrow 1}\left(1-\rho^{2}\right)^{1 / 2} \partial \chi / \partial z=2\left\{\pi P_{2 m}(0)\right\}^{-1} .
$$

Suppose $V$ is an axially-symmetric harmonic function defined in $z \geqslant 0$ subject to the boundary conditions on $z=0$,

$$
V=f(\rho) \text { for } 0 \leqslant \rho \leqslant 1, \quad \partial V / \partial z=0 \text { for } \rho>1 .
$$

Then using the representation

$$
f(\rho)=\sum_{0}^{\infty} a_{n} P_{2 n}\left\{\left(1-\rho^{2}\right)^{1 / 2}\right\} \quad \text { for } 0 \leqslant \rho \leqslant 1,
$$

where

$$
\begin{aligned}
a_{n} & =(4 n+1) \int_{0}^{1} f(\rho) P_{2 n}\left\{\left(1-\rho^{2}\right)^{1 / 2}\right\} \frac{\rho}{\left(1-\rho^{2}\right)^{1 / 2}} d \rho \\
& =(4 n+1) \int_{0}^{1} f\left\{\left(1-\eta^{2}\right)^{1 / 2}\right\} P_{2 n}(\eta) d \eta,
\end{aligned}
$$

the potential $V$ may be expressed as a series of solutions of the type (27). If $f(\rho)$ is a polynomial in $\rho^{2}$ the constants $a_{n}$ may be found using (2, Table 22.9).

Alternatively they may be evaluated using the Gauss integration formula (2, 25.4.36). The representation (31) is the expansion in Legendre polynomials over the range $-1 \leqslant \eta \leqslant 1$ of the even function defined by $f\left\{\left(1-\eta^{2}\right)^{1 / 2}\right\}$ over the range $0 \leqslant \eta \leqslant 1$.

Problem B. On $z=0, \frac{\partial \psi}{\partial z}=\frac{P_{2 n+1}\left\{\left(1-\rho^{2}\right)^{1 / 2}\right\}}{\left(1-\rho^{2}\right)^{1 / 2}}$ for $0 \leqslant \rho<1$,

$$
\psi=0 \text { for } \rho>1 \text {. }
$$

Solution: $\quad \psi=\frac{P_{2 n+1}(\eta) Q_{2 n+1}(i \xi)}{i Q_{2 n+1}^{\prime}(+i 0)}=\frac{2 P_{2 n+1}(\eta) Q_{2 n+1}(i \xi)}{\pi(2 n+1) P_{2 n}(0)}$.

$$
\begin{gathered}
\text { On } z=0, \psi=-\frac{2 P_{2 n+1}\left\{\left(1-\rho^{2}\right)^{1 / 2}\right\}}{\pi(2 n+1)^{2}\left\{P_{2 n}(0)\right\}^{2}} \quad \text { for } 0 \leqslant \rho<1, \\
\frac{\partial \psi}{\partial z}=\frac{2}{\pi} \frac{1}{\left(\rho^{2}-1\right)^{1 / 2}} Q_{2 n+1}\left\{i\left(\rho^{2}-1\right)^{1 / 2}\right\}, \quad \rho>1 .
\end{gathered}
$$

Note that on $z=0$,

$$
\lim _{\rho \rightarrow 1}\left(\rho^{2}-1\right)^{1 / 2} \frac{\partial \psi}{\partial z}=-\frac{2}{\pi(2 n+1) P_{2 n}(0)} .
$$

Suppose $W$ is an axially-symmetric harmonic function defined in $z \geqslant 0$ subject to the following boundary conditions on $z=0$ :

$$
\partial W / \partial z=g(\rho) \text { for } 0 \leqslant \rho \leqslant 1, \quad W=0 \text { for } \rho>1 .
$$

If $\left(1-\rho^{2}\right)^{1 / 2} g(\rho)$ has the representation given by

$$
\eta g\left\{\left(1-\eta^{2}\right)^{1 / 2}\right\}=\sum_{n=0}^{\infty} b_{n} P_{2 n+1}(\eta), \quad 0 \leqslant \eta \leqslant 1,
$$


then $W$ may be expressed as a series of solutions of type (32). The representation (36) is the expansion in Legendre polynomials over $-1 \leqslant \eta \leqslant 1$ of the odd function defined by $\eta g\left\{\left(1-\eta^{2}\right)^{1 / 2}\right\}$ over $0 \leqslant \eta \leqslant 1$.

If $g(\rho)$ is a polynomial in $\rho^{2}$, the constants $b_{n}$ may be found by using $(2$, Table 22.9). Alternatively

$$
\begin{aligned}
b_{n} & =(4 n+3) \int_{0}^{1} \eta g\left\{\left(1-\eta^{2}\right)^{1 / 2}\right\} P_{2 n+1}(\eta) d \eta \\
& =(4 n+3) \int_{0}^{1} \rho g(\rho) P_{2 n+1}\left\{\left(1-\rho^{2}\right)^{1 / 2}\right\} d \rho .
\end{aligned}
$$

This integral may be evaluated numerically using a Gauss integration formula such as (2, 25.4.34). These solutions have been given by Tranter (6).

In both problems $A$ and $B$ it is probable that the quantities of interest are the 'unknown' boundary values on $z=0$. Over the range $0 \leqslant \rho \leqslant 1$ these depend on $P_{n}\left\{\left(1-\rho^{2}\right)^{1 / 2}\right\}$ and over the range $\rho \geqslant 1$ these depend on $Q_{n}\left\{i\left(\rho^{2}-1\right)^{1 / 2}\right\}$. The associated singularities in the normal derivaties at $\rho=1$ are given above together with the appropriate multiplicative factors. The Legendre polynomials $P_{n}(t)$ are tabulated in (2, Table 8.1). The function $Q_{n}($ it $)$ does not appear in most standard tables. It may be shown that

$$
\begin{aligned}
& i Q_{0}(i t)=\tan ^{-1}\left(\frac{1}{t}\right), \\
& Q_{1}(i t)=-1+t \tan ^{-1}\left(\frac{1}{t}\right) .
\end{aligned}
$$

The following recurrence relation is given in $(2,8.5 .3)$ :

$$
(n+1) Q_{n+1}(z)=(2 n+1) z Q_{n}(z)-n Q_{n-1}(z)
$$

for complex values of $z$. From these relations it is possible to generate $Q_{n}$ (it) by a straightforward numerical procedure.

\section{Discussion}

The solutions presented in this note stem from the method of separation of variables in elliptical and ellipsoidal coordinate systems. These solutions have been used by Gladwell and England (4) in the solution of some crack and punch problems in elasticity. England and Shail (7) have recently examined these solutions in more detail and considered the relation between these methods and integral equation techniques for the solution of mixed boundary-value problems. This note has been written in an attempt to collate these solutions with reference to Laplace's equation. The two-dimensional solutions given in $\$ 2$ do not appear to be well-known. However Tricomi (8) has derived solutions (22), (23) of the Hilbert integral equation by an alternative method but comments that these relations may be deduced from the analytic functions (1) and (2). A referee has drawn my attention to Pykhteev (9). In this paper and in two earlier works referred to in (9), Pykhteev considers solutions of Hilbert integral equations in particular deriving the representations (24) and (25). His 
attention is concentrated on expansions of the type $\Sigma_{s=1}^{N} \alpha_{2 s-1} \phi_{2 s-1}(z)$ where $\phi_{s}(z)=$ $\Sigma_{n=2}^{\infty} R_{2 n-1}(z) /(2 n-1)^{s}$ in the notation used in this paper. These expansions are rather more complicated than those suggested earlier in this paper and the reasons for their adoption are not explained. Pykhteev also uses the definition $U_{n}(x)=\sin n \theta$ rather than the more common $U_{n}(x)=\sin (n+1) \theta / \sin \theta$, where $x=\cos \theta$, which affects certain formulae.

Erdogan, Gupta and Cook (3) have used Gaussian integration formulae to develop a numerical technique for the solution of integral equations with singular parts of the form

$$
\int_{-1}^{1} \frac{w(t)}{t-x} f(t) d t
$$

in which $w(t)$ is a known weight function and $f(t)$ the unknown function. Their method is to choose the related polynomials to be orthogonal with respect to the weight function $w(t)$ over the interval $(-1,1)$. In the case of the problems of $\$ 2$ the corresponding integral equations have the weight functions $\left(1-t^{2}\right)^{ \pm 1 / 2}$ and the resulting polynomials are the Chebyshev polynomials (which arise naturally from the separation of variables technique). Some of the special solutions given in (4) and this note may be derived from (3) but these solutions are not quoted in (3).

The corresponding use of oblate spheroidal coordinates in three-dimensional problems is relatively well-known and the axially-symmetric solution $P_{n}(\eta) Q_{n}(i \xi)$ is given in several texts. Sneddon $(1$, p. 69) comments that it is a fairly difficult matter to compute the field quantities at any point whose Cartesian coordinates are prescribed, but, at points on $z=0$, the calculation of the potential or its normal derivative would seem relatively straightforward using the tables given in (2) and the recurrence relation to define $Q_{n}(i \xi)$.

\section{REFERENCES}

(1) I. N. SNeddon, Mixed Boundary Value Problems in Potential Theory (North-Holland, 1966).

(2) M. Abramowitz and I. A. Stegun, Handbook of Mathematical Functions (Dover, 1964).

(3) F. ERdogan, G. D. GuPTA and T. S. Cook, Numerical solution of singular integral equations, Mechanics of Fracture, Vol. 1 (Noordhoff, 1973, Ed. G. Sih), 368. 175.

(4) G. M. L. GladWell and A. H. EnGland, Quart. J. Mechs. Appl. Maths, 30 (1977), 1968).

(5) L. Fox and I. B. PARker, Chebyshev Polynomials in Numerical Analysis (Oxford,

(6) C. J. Tranter, Quart. J. Math. 1 (1950), 1.

(7) A. H. England and R. Shail, Quart. J. Mechs. Appl. Maths. 30 (1977), 397.

(8) F. G. TRICOMI, Integral Equations (Interscience, 1957), §4.3.

(9) G. N. PYKHTEEv, Problems of Continuum Mechanics, S.I.A.M. (1961), 350.

Department of Theoretical Mechanics

UNIVERSITY OF NOTTINGHAM 